\title{
Integrating Earthwork Ontology and Safety Regulations to Enhance Operations Safety
}

\author{
A. Taher ${ }^{\text {a }}$, F. Vahdatikhaki ${ }^{\text {b }}$, and A. Hammad ${ }^{\mathrm{c}}$ \\ ${ }^{a}$ Concordia University, Concordia Institute for Information Systems Engineering, (CIISE), Canada \\ ${ }^{b}$ University of Twente, Department of Construction Management and Engineering, The Netherlands \\ ${ }^{\mathrm{c} C o n c o r d i a}$ University, Concordia Institute for Information Systems Engineering, (CIISE), Canada

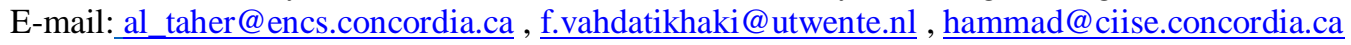

\begin{abstract}
-
Safety in the construction industry remains a major challenge despite the technological advancements made in recent years. In recent years, ontologies are applied to give a formal structure to the knowledge in different domains. Ontologies also facilitate the integration of various domain knowledge and thus allow for better cross-functional developments (e.g., operator support systems that consider safety and productivity at the same time). The authors have previously developed a comprehensive Earthwork Ontology (EW-Onto). However, there has been no linkage between safety regulations and EW-Onto. Therefore, this research aims to: (1) develop a formal representation of earthwork safety regulations knowledge, and (2) integrate this knowledge with EW-Onto. A case study is developed to validate the integrated ontology. It is shown that the integrated ontology can be used to bridge the gap between high-level safety regulations and task-level instructions.
\end{abstract}

\section{Keywords -}

Earthwork; Ontology; Regulations; Safety

\section{Introduction}

Worker and equipment safety is a critical issue in the construction domain. Numbers from construction sites show that hazards in workzones affect projects at different levels. According to OSHA [1], in 2016, 21.1\% of fatalities in the private industry are related to construction. Ignoring the safety rules, not following safety regulations, weather, and reckless equipment operators are the main factors that lead to accidents on construction sites. Accidents in construction sites are the main reason for the loss of life, which in turn affects schedules, productivity, costs, and the reputations of construction firms. In earthwork domain, excavation, especially trenches, are recognized as the most hazardous construction operation that can lead to serious accidents, such as cave-ins, toxic atmospheres, and falls [1]. Job Hazard Analysis (JHA) from OSHA [2], is a technique used for identifying, evaluating, and controlling these types of hazards. This technique is one of the different methods used for checking if the variables that are related to workers, tools, equipment, and the environment meet the associated regulations and rules. The data collected from construction sites using different technologies can enhance construction site productivity and safety. Moreover, combining human (e.g., workers, operators, designers, and coordinators) experience and best practices that are gained from previous similar projects is another method to avoid accidents in workzones. However, it is necessary to link the related hazards at the different levels of the project with other entities on the site (e.g., products, equipment, and the surrounding environment) in order to have better decision making. In recent years, ontologies are applied to give a formal structure to the knowledge in different domains. Ontologies also facilitate the integration of various domain knowledge and thus allow for better crossfunctional developments (e.g., operator support systems that consider safety and productivity at the same time). The authors have previously developed a comprehensive Earthwork Ontology (EW-Onto). However, there has been no linkage between safety regulations and EW-Onto. Therefore, this research aims to: (1) develop a formal representation of earthwork safety regulations knowledge, and (2) integrate this knowledge with EWOnto.

The rest of this paper is organized as follows: Section 2 discusses the related works on the safety regulations in construction. The methodology considering the different hazards in earthwork are presented in Section 3. Section 4 discusses the implementation of the ontology. Section 5 presents the conclusion and future work. 


\section{Related works}

\subsection{Safety Checking Methods and Techniques in Construction}

Providing and performing safety procedures in workzones are a major factor for the success of the construction industry. These procedures should be followed by everyone involved in the business, which, in turn, saves lives and reduces the costs of business. Safety checking, which is the main part of safety management, aims to identify potential hazards before they occur on construction sites. Different methods and techniques are used to perform safety checks on construction workzones. The most efficient method is JHA, which defines the relationships between jobs, tools, workers, and the surrounding environment and provides a list of procedures and resources that are needed to prevent or reduce these hazards [2]. Occupational Risk Assessment (ORA) [3] is a process that is performed on the construction site to gather information from different sources to build better knowledge about hazards [4]. Check-list [5] technique is used to perform ORA on construction sites to define the safety issues at early stages of the work. Zhang, et al. [6] outlined a framework for early hazards identification in the construction model by integrating the Building Information Model (BIM) and safety regulations. Thus, the related hazards and the corresponding prevention procedures can be automatically identified and applied. 4D-BIM technologies connect the safety activities, construction planning, and visualizing the safety arrangements at any time [7]. Thus, the safety personnel and the designer with less knowledge about safety can use BIM-based friendly software to organize the Safety Knowledge (SK) and to improve occupational safety.

\subsection{Ontological Modeling in Construction}

Ontologies aim to represent the implicit knowledge in a domain in an explicit way through an organized structure of related concepts and relationships. Gruber [8] defined the ontology as "an explicit specification of a conceptualization."

Different studies use ontology and combine it with modeling techniques (e.g., BIM) [6][9]. Lee et al. [9] proposed an ontological approach for quantity take-off using BIM as a data source. The developed ontology is then used to infer the suitable items based on the estimated cost. Zhong et al. [10] provided an approach to integrate construction processes with regulation, which are related to quality compliance.

Zhang et al. [11] described an approach to store and re-use the safety management knowledge in construction. This approach links three main models, which are represented as ontology (i.e., product model, process model, and safety model). Ding et al. [12] proposed an approach to combine BIM with ontology to semantically organize construction risk knowledge. Wang and Boukamp [13] created a framework to improve access to the company's JHA. The framework uses the ontology to organize knowledge about activities, jobs' steps, and the related hazards.

Earthwork operations are a common part of construction projects. These operations include excavation, hauling, dumping, scraping, and grading. Earthwork operations account for $20 \%$ of the total cost of road building projects [14]. Using advanced information technologies in earthwork operations to improve safety and productivity is a major concern for researchers. Vahdatikhaki et al. [15] proposed the application of $a$ Multi-Agent System (MAS) as a means to support fleetlevel coordination for earthwork operations. Ontologies are one of the advanced technologies that have been used in construction to facilitate not only the human-to-human but also machine-to-machine communications by formalizing the information exchange scheme [16]. Taher et al. [16] proposed a framework to develop Earthwork Ontology (EW-Onto) to support and enhance the communication and data exchange between different stakeholders in earthwork projects.

\subsection{Ontology Development Methodologies}

The foundation Ontology (FO), also known as toplevel or upper ontology, is an ontology that describes the most general terms across the domains. Zhou et al. [17] explained that certain studies might include some steps from the previous methodologies for developing their ontologies. In the 1990's, some methodologies for developing ontologies have been outlined. Skeletal methodology [18], and METHONTOLOGY [19] are examples of general methodologies used to build ontologies. IDEF5 is an ontology capture method and one of the Integrated DEFinition (IDEF) family languages that support the analysis and design of models [20]. Other methodologies or approaches are used to build the ontologies by re-using the existing ontologies or integrating two or more ontologies.

Description Logics (DL) is a language to formalize the knowledge representation that provides high-level descriptions of the world to be used in intelligent systems [21]. DL delivers syntax to describe the knowledge by expressions, which are built as atomic concepts, atomic roles and role constructors. DL is separated into three formalism components: Terminological Component (TBox), Assertion Component (ABox), and Role Axiom (RBox). TBox axioms describe the general properties of concepts and contain the intensional knowledge in the form of taxonomy or terminology such as concept inclusion. ABox axioms contain the assertional knowledge for specific individuals in the domain. 
Whereas RBox refers to the properties of roles, such as role equivalence axioms and role inclusion [22].

\section{Proposed Framework}

The framework used to develop EW-SKB follows the approach of METHONTOLOGY for the following reasons: (1) it is application-independent and mature; (2) it is well-documented and has clear development activities; (3) it is based on the experience acquired from developing ontologies for many domains [19]; and (4) it has an integration step, which facilitates reusing EWOnto as the main base of EW-SKB.

\subsection{Main Steps to Develop EW-SKB}

The best practices and knowledge in the earthwork domain and the related safety issues are used in EW-SKB development. Figure 1 illustrates the main phases of developing EW-SKB as explained in detail below.

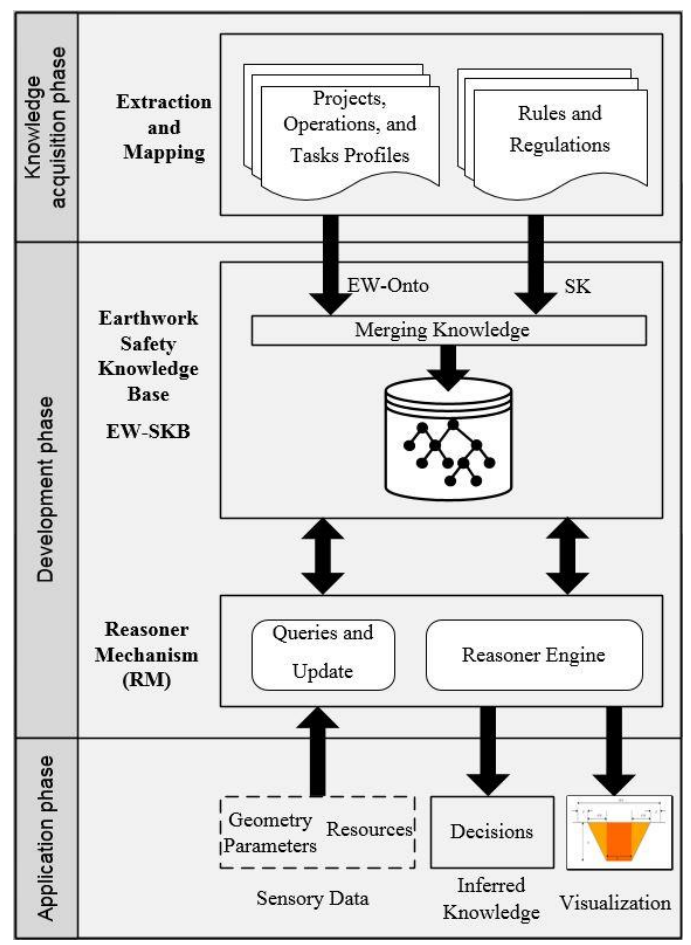

Figure 1. Proposed framework

\subsubsection{Knowledge Acquisition Phase}

The initial phase of the proposed framework, i.e., the knowledge acquisition phase, consists of defining the scope of the EW-SKB and defining concepts and taxonomies based on the requirements. The requirement data (e.g., terms and properties) are collected from various sources, such as textbooks and online resources (e.g., OSHA). EW-SKB should address the Competency Questions (CQ), such as: Why do we need to develop or extend the ontology? What are the domains and the scope of the ontology? Who are the users of the ontology? Answering these questions addresses parts of the requirements in the earthwork safety domain. Also, it helps to clarify what should be included in the developed ontology, and to which level of detail it should be developed. Furthermore, the basic concepts and relationships of EW-SKB structure is defined at this phase. The concepts, terms, properties, and instances are parts of the Glossary of Terms (GT). Developing the GT becomes easier when the specifications are clear.

\subsubsection{Development Phase}

In this phase, safety knowledge is added to the EWOnto. SK includes the related concepts and relationships that link EW-Onto, related hazards, and related rules and regulations. The development phase includes the steps to add the SK to EW-Onto. The formalization of EW-SKB starts with transferring the conceptual model into the formal model using DL. Table 1 illustrates some examples of the conceptual components represented as terminology, assertion, and rules axioms, which define the concepts, individuals, and relationships, respectively. These components are added to the EW-Onto to link the concepts to soil types, product hazards, and equipment hazards. The Reasoning Mechanism (RM) or reasoner engine is the mechanism that represents the inferred knowledge and performing the queries over the explicit knowledge in the ontology. The RM checks the consistency of the developed ontology and validates the update of the relationships to ensure that there is no conflict.

\subsubsection{Application Phase}

This phase consists of any changes and updates of EW-SKB. The assumption here is that the ontology receives the sensory data from the site, including geometry data and resources data. Geometry data is more about the terrain model and the products (e.g., depth, width). Resources data includes data about the different types of resources in the earthwork domain including equipment (e.g., hoes, trucks), and materials (e.g., soil) from the workzones. The decisions are then generated based on the received data and the rules in EW-SKB. The visualization mechanism is not included in this paper and will be considered in future work.

\subsection{Earthwork Related Hazards}

In earthwork operations, there are a variety of hazards that affect the earthwork project at different levels. In this research, and as one of the main steps to map the concepts in the first phase, hazards in earthwork operations are classified into three main categories: material hazards, product hazards, and equipment hazards. 
Table 1. Examples of TBox, ABox, and RBox components related to soil classification, product hazards, and equipment hazards

\begin{tabular}{c|c|c}
\hline Axiom & Explanation & Examples \\
\hline TBox & $\begin{array}{c}\text { Express the terminological } \\
\text { knowledge }\end{array}$ & $\begin{array}{c}\text { SoilType_A, HazardWorkzone, ProtectionSystem, SlopingSystem, } \\
\text { CaveIn, Path, Segment, Intersection, ExcavationZone, DumpingZone, } \\
\text { IntersectionZone, WarningRange }\end{array}$ \\
\hline ABox & $\begin{array}{c}\text { Express the knowledge } \\
\text { about the individuals }\end{array}$ & $\begin{array}{c}\text { Soil1, Soil2, Workzone1, TrenchBox1, Slop1, Path1, Segment3, } \\
\text { IntersectionPointT1, ExcavationZone }\end{array}$ \\
\hline RBox & $\begin{array}{c}\text { Describe the properties of } \\
\text { the roles }\end{array}$ & $\begin{array}{c}\text { hasStructure, hasTexture, hasType hasSiltAndClayPercentage, } \\
\text { hasSoilType, hasHazard, hasSlopAngle, hasSpeed, hasLocation, } \\
\text { hasCollisionWarning, hasToStop, hasDirection }\end{array}$ \\
\hline
\end{tabular}

It should be noted that products (e.g., trench) and materials (e.g., soil) are linked to each other. Product hazards are the result of the geometry of the product (e.g., length, width, and depth). Whereas, soil hazards are linked with the properties of the soil. Different actors who share and perform the processes and tasks (e.g., equipment operators, and on-feet workers) need to avoid hazards during the project at any level. To make the situations at the site more practical, the excavations and trenches are divided into workzones. Thus, each trench is composed of some workzones that may be different in their properties (e.g., soil type, depth, or other nearby workzones). Moreover, at each workzone, the tasks are performed by one individual or a team of different equipment and workers.

\subsubsection{Material Hazards}

In this research, and since the earthwork operations are linked to soil, the hazards related to soil are considered. These hazards are related to the different materials that are used in the project in different earthwork operations, such as cleaning and grabbing operations, excavation operations, and compaction operations. In some earthwork projects, explosive materials could be used in cleaning and grubbing operations, which affect the soil (e.g., the stability).

Soil classification plays a vital role in safety regulations, where each type of soil has its own structure and properties, which affect soil behavior under different circumstances. Soil classification can be obtained based on different values of the properties [23], which directly affect the stability of the excavation and the choice of suitable safety procedures and resources. OSHA standard number 1926 Subpart P Appendix A [24] provides examples of the guidelines that can be applied to classify the soil based on various properties. In EW-SKB, the quantitative properties are used to write the classification rules, which are: the structure of the soil (i.e., cohesive, granular, or granular cohesionless); the percentage of silt and clay in the soil, this percentage can be less or more than 15\%; and the Unconfined Compressive Strength value (UCS), which is a result from the soil lab test and signifies the consistency and measures shear strength.

Different OSHA standard rules are translated to Semantic Web Rule Language (SWRL) to integrate them into the developed ontology. The next paragraph lists examples of SWRL in EW-SKB to classify the soil based on these quantitative properties. In addition, the pseudocode illustrates the logical steps to apply the rule. Soil classification is used to link to other rules related to the product hazards

Rule 1. This rule uses the structure of the soil, the clay percentage, and the UCS value to classify the soil and determine the soil texture.

Start

Input: The soil structure, the Silt and Clay percentage (scp), The value of (ucs)

Output: Soil classification, The soil texture

For each Soil sample do

If Soil has structure = "Cohesive" and scp $>15 \%$ and ucs $>1.5$ and has plasticity and is fissured

Set soil has type (A)

Set soil has texture (clay)

end if

end

End

Soil(?s)^hasStructure(?s,"Cohesive")^hasSiltAndClayPe rsentage(?s,?scp)^swrlb:greaterThan(?scp,0.15)^hasUnc onfinedCompressiveStrengthValue(?s,?ucs)^swrlb:great erThan(?ucs, 1.5)^hasPlasticity(?s,true $)^{\wedge}$ isFissured(?s,fal se)->Soil_Type_A(?s)^ hasType(?s,"A")^hasTexture (?s, "Clay")

\subsubsection{Products Hazards}

Products hazards describe the hazards that are linked to the final or intermediate outcomes of the processes and tasks, such as trenches, excavations, and holes. The focus here is on the trench-related hazards and how to improve the safety level in this type of earthwork.

OSHA rule number 1926.652, subpart $P$ [25] is related to requirements for protective systems for 
excavation. This clause specifies in general the rules related to trench shields. OSHA regulation (1926 Subpart P App B) [26] states the angle of slope for each type of soil. Based on these regulations, the following examples of SWRL rules link the workzones, the type of the earthwork operation, the type of the soil, the expected hazards, and how hazards can be avoided by performing certain procedures.

Rule 2. This rule checks the depth of the workzone. If the depth is more than $153 \mathrm{~cm}$, then this workzone is classified as hazard workzone. The rule determines the type of hazard that this workzone has and which safety resources are needed to make it safer. As a result, the excavation operation is classified as hazard operation.

\section{Start}

Input: The workzone depth (d)

Type of the earthwork operation (exc)

Output: The potential hazard

The safety resource needed

Classification of the workzones

For each excavation operation do

For each workzone in the operation do

If $\mathrm{d}>152 \mathrm{~cm}$

Set workzone has hazard (CaveIn)

Set workzone needs safety resource

(ProtectionSystem)

Set workzone is hazard workzone

Set excavation operation has hazard

$$
\text { end if }
$$

end

end

End

ExcavationOperation(?exc)^ProtectionSystem (?ps)^ CaveIn(?ca $)^{\wedge}$ swrlb:greaterThan(?d,152)^has Depth (?w,?d)^hasWorkzone(?exc,?w)^Workzone(?w)-> hashazard(?w,?ca)^needSafetyResource(?w,?ps)^ HazardWorkzone(?w)^has (?exc, Hazard)

Rule 3. This rule classifies the earthwork operation based on the range of the depth and the soil type. If the depth of the operation in the range between $365 \mathrm{~cm}$ and $609 \mathrm{~cm}$, and the soil type is A, then this operation is classified as hazard operation. The hazard type of this operation is Cave In hazard. Thus, this operation needs a safety procedure, which in this case is making a slop with $53^{\circ}$.

Start

Input: The workzone depth (d), Type of Soil, and

Type of the earthwork operation (exc)

Output: The potential hazard, The safety procedure needed, Classification of the earthwork operation

For each excavation operation do

If $\mathrm{d}>365 \mathrm{~cm}$ and $<609 \mathrm{~cm}$ and Soil $\quad$ Type $=A$

Set excavation operation needs safety procedure (Trench Slop)
Set excavation operation needs slop Angle $53^{\circ}$

Set excavation operation has hazard (CaveIn)

Set excavation operation is hazard operation end if

end

End

SlopingSystem(?ss $)^{\wedge}$ Workzone $(? w)^{\wedge}$ Excavation Operation(?exc)^hasWorkzone (?exc,?w) $)^{\wedge}$ hasType $(? \mathrm{~s}, " \mathrm{~A} ")^{\wedge}$ CaveIn(?ca $)^{\wedge}$ hasDepth $(\mathrm{w}, ? \mathrm{~d})^{\wedge}$ swrlb:greater Than (?d,365)^swrlb:lessThan (?d,609)^hasSoilType $(? \mathrm{w}, ? \mathrm{~s})^{\wedge}$ Soil $(? \mathrm{~s})->$ needSafetyProcedure $(? \mathrm{exc}, ? \mathrm{ss})^{\wedge}$ needSlopAngle(?exc,53)^hashazard (?exc,?ca)^Hazard Operation (?exc)^has(?exc,Hazard)

The steps to identify the hazards in the earthwork workzones and equipment are illustrated in Figure 2. The algorithm considers the hazards in workzones, which are a combination of soil and product hazards.

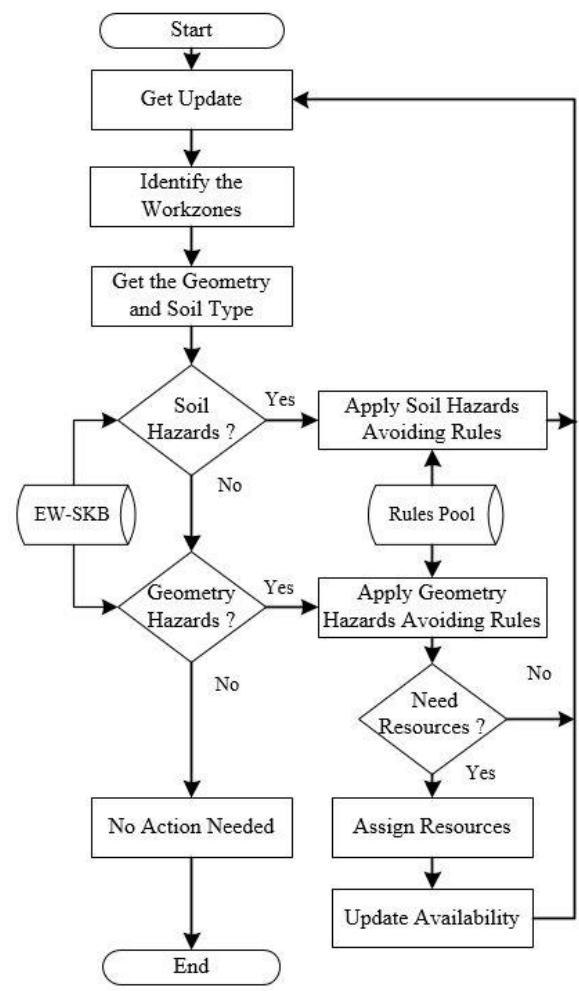

Figure 2. Material and product hazard identification

The steps begins with retrieving an update of a particular workzone. The type of workzone is identified (e.g., excavation or trench), followed by retrieving the geometry and soil type of the workzone. Then, the type of hazards are recognized based on applying the rules of the ontology. In the case that resources are needed to avoid the hazard (e.g., trench boxes), the assigned resources are used to update the availability of resources. 
Then, an update is applied to ensure that the safety rules will not be triggered again.

\subsubsection{Equipment Hazards}

Several types of equipment (e.g., trucks, scrapers, dozers and hoes) are used in earthwork sites because of their ability to perform different tasks. These pieces of equipment are usually working together as a team to achieve the work. Equipment hazards could be called the operation hazards, where the risks are higher when several teams of equipment perform different tasks in parallel in a congested site. For equipment hazards, trucks are considered as sources of hazards when they are moving from one location to another. There are a variety of hazards related to trucks, which can increase the potential hazards of accidents and collisions, such as exceeding the maximum load or speed on roads and paths, and right-of-way regulations. Uncontrolled paths in construction sites pose high potential hazards to equipment and workers. Intersections have more complex traffic situations, which are influenced by a variety of safety rules, such as the permitted and prohibited directions and different priorities for equipment crossing the intersection at the same time. Moreover, the hazard level is raised when the equipment is operated by reckless drivers or poorly-trained operators. However, using a decision-support mechanism can help the operators in improving their safety.

Figure 3 illustrates how the hazards related to equipment are identified after retrieving the update of sensory data from the site.

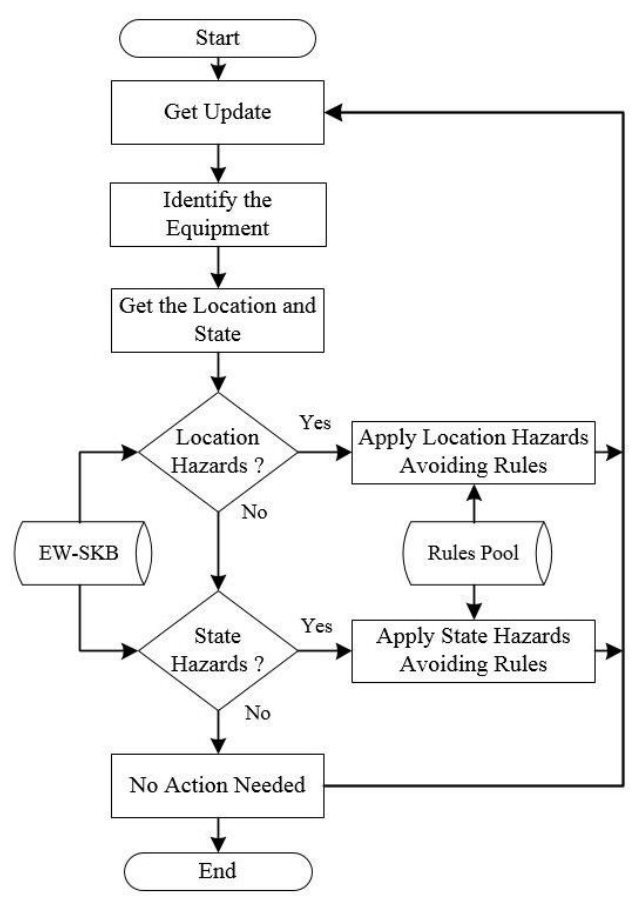

Figure 3. Equipment hazards identification
Generally, the equipment hazards are divided into two main categories, namely hazards related to equipment location and hazards related to the equipment state. Hazards related to equipment location are the result of changes in locations while the equipment is preforming a task (e.g., a truck or scraper moving between excavation and dumping area) or equipment relocation (e.g., a hoe moving from one workzone to another). Whereas the hazards related to the equipment state are the result of performing the tasks (e.g., a hoe swinging to the digging area). The hazards related to the equipment state are beyond the scope of this paper.

Data properties can obtain their values from sensors attached to the equipment (e.g., truck) and/or located on the paths and intersections. locatedAt, movingFrom, goingTo, isOn, isUnder, and isToward are object properties linked the individuals. Figure 4 shows a hazards situation where a group of three trucks performs their tasks (i.e., hauling and return from the dumping zones).

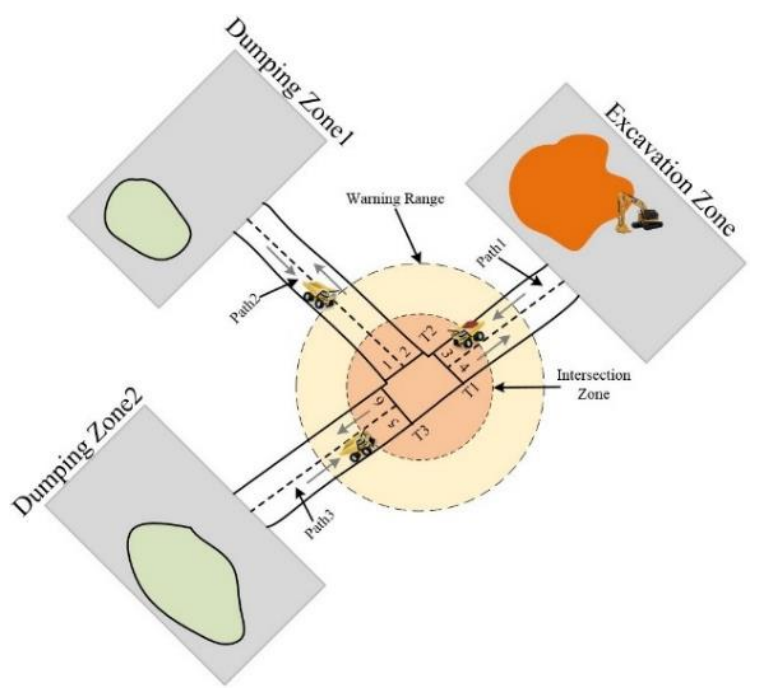

Figure 4. The case study scenario of the uncontrolled intersection

The trucks equipment are located near an uncontrolled intersection. This scenario simulates the hazards related to the equipment locations. Figure 4 shows the simulated scenario. Trucks on paths $(1,2$, and 3 ) are moving in different directions. Each truck is labeled based on its direction and its path. SWRL is used to link the above-mentioned concepts with other concepts and entities in EW-Onto. SWRL is also used in EW-SKB to infer the safety decisions and create a safe driving environment, such as the right-of-way, sending a warning, order to stop, or order to slow down. The next SWRL rules are examples that formularize the knowledge about truck hazards at an uncontrolled intersection.

Rule 4. This rule illustrates an example of the orders that 
trucks are received based on the situation of truck with the priority. In this rule, Truck1 has the priority, its direction is straight, and is located at the intersection. Thus, Truck3, which its direction is left receives the order to stop in case it is under the warning ranges.

\section{Start}

Input: The location, The direction, Truck name

Output: The order to stop or slow down

For each Truck do

If label = "Truck1" and location= isLocatedAt

(IntersectionPointT1) and direction= "GoingStraight"

If label= "Truck3" and direction= "GoingLeft" and location =isUnder (Warning_Range)

Set Truc
end if
end if
end
End

Truck(?tr1)^hasLabel(?tr1,"Truck1")^isLocatedAt(?tr1, IntersectionPointT1 $)^{\wedge}$ hasDirection(?tr1,"GoingStraight" )$^{\wedge} \operatorname{Truck}(? \operatorname{tr} 3)^{\wedge}$ hasLabel(?tr3,"Truck3")^hasDirection(?tr 3,"GoingLeft")^isUnder?tr3,?wr)^Warning_Range(?wr) -> hasToStop(?tr3, true)

\section{Implementation and Case Study}

As mentioned in Section 1, EW-Onto is used as the main part of EW-SKB. In this paper, Protégé, 5.0.2 [27] is used to edit EW-Onto by adding the related concepts and relationships using a set of graphical plugins. Moreover, SWRL rules can be added and edited using Protégé. The verification is done by checking whether EW-SKB can answer the CQ or not. Furthermore, the consistency of EW-SKB is checked using a reasoner (HermiT reasoner). Figure 5 illustrates an example of using the reasoner engine based in Rule 1 to define the soil type.

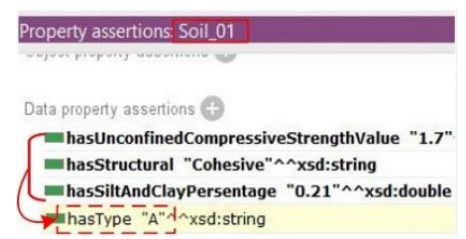

Figure 5. Reasoning engine result for soil classification

Thus, Soil_01 is soil type A based on the values of this sample. Figure 6 provides examples of hazard related to workzone and excavation operation based on the Rules 2 and 3. Excavation-100-01 has inferred a hazard because it has an instance of workzone (i.e., Workzone001), and has soil type A.

A simulated case study is conducted to verify the developed implementation. As demonstrated in Figure 4 and based on Rule 4, Figure 7(a) shows the simulated scenario. Truck1 isOn Path1, and isToward the intersection. This truck gets the priority (hasPriority: true) because it is located in the intersection area and is loaded (isLoaded: true).

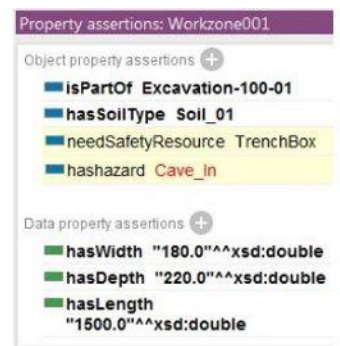

(a)

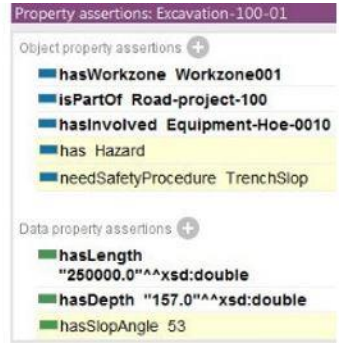

(b)
Figure 6. Reasoning engine results for workzones (a) and for excavation operation (b)

The other trucks (Truck2 and Truck3) are ordered to stop or slow down. The messages to the other trucks are based on their locations and directions. For example, as shown in Figure 7(a), Truck1 is moving in a straight direction. Thus, Truck2 received the order to stop (hasToStop=true) as in Figure 7(b) and Truck3 received the order to slow down (hasToSlow=true) because its direction is also going straight (hasDirection"GoingStright") as shown in Figure 7(c). Whereas the order will be changed to stop (hasToStop=true) if Truck3 direction is going left (hasDirection" GoingLeft") as shown in Figure 7 (d).

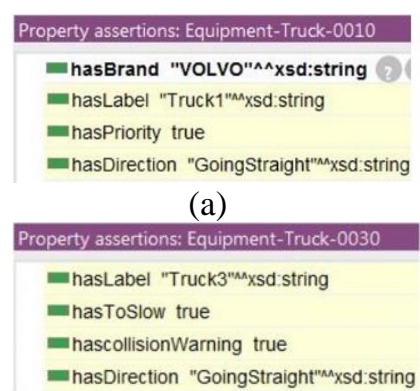

(b)

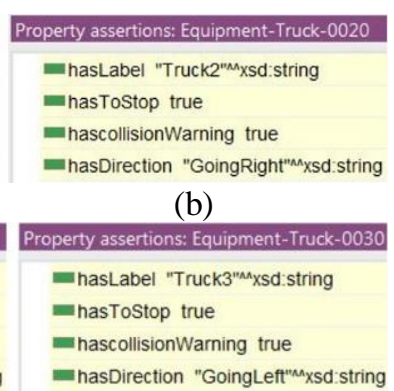

(d)
Figure 7. Reasoning engine results in EW-SKB for trucks at an uncontrolled intersection.

\section{Conclusion and Future Work}

In this paper, the previously developed earthwork ontology (EW-Onto), has been augmented by adding safety knowledge base (EW-SKB). EW-SKB is a combination of essential knowledge related to the safety issues in earthwork, which can contribute to improving safety decision-making. Knowledge associated with safety is integrated with EW-Onto to link the different concepts in EW-SKB. EW-SKB has the added value of integrating different entities (e.g., equipment, trench, and 
soil) in construction sites with safety regulations. The potential benefits of the developed ontology are combined from the scalability nature and the automatic inferring decisions that can be taken based on data collected from actual projects. The future work will focus on developing an approach to visualizing the decisions and combining soil and sensor ontologies, which will add a more robust base to extend EW-SKB. Thus, more comprehensive representation of the concepts and relationships will be included in the resulting ontology.

\section{References}

[1] OSHA. Commonly used statistics, On-line: www.osha.gov/oshstats/commonstats.html, Accessed: 27/03/2018.

[2] Chao E. L. and Henshaw J. L. Job Hazard Analysis (JHA), OSHA Publication 3071(Revised). Department of Labour, (OSHA), 2002.

[3] Pinto A., Nunes I. L. and Ribeiro R. A. Occupational risk assessment in construction industry-Overview and reflection. Saf.Sci., 49(5): 616-624, 2011.

[4] Lu Y., Li Q., Zhou Z. and Deng Y. Ontology-based knowledge modeling for automated const. safety checking. Saf.Sci., 79: 11-18, 2015.

[5] Mattila M., Rantanen E. and Hyttinen M. The quality of work environment, supervision and safety in building const. Saf.Sci., 17(4): 257-268, 1994.

[6] Zhang S., Teizer J., Lee J., Eastman C. M. and Venugopal M. Building information modeling (BIM) and safety: Automatic safety checking of construction models and schedules, Auto. Const., 29: 183-195, 2013.

[7] Kiviniemi M., Sulankivi K., Kähkönen K., Tarja M. and Merivirta M. BIM-based safety management and communication for building construction., VTT Rese. Notes 2597, 2011.

[8] Gruber T. R. A translation approach to portable ontology specifications. Knowledge acquisition, 5(2): 199-220, 1993.

[9] Lee S., Kim K. and Yu J. BIM and ontology-based approach for building cost estimation. Auto. Const., 41: 96-105, 2014.

[10] Zhong B., Ding L., Luo H., Zhou Y., Hu Y. and Hu H. Ontology-based semantic modeling of regulation constraint for automated construction quality compliance checking. Auto. Const., 28: 58-70, 2012.

[11] Zhang S., Boukamp F. and Teizer J. Ontologybased semantic modeling of construction safety knowledge: Towards automated safety planning for job hazard analysis. Auto. Const., 52: 29-41, 2015.

[12] Ding L., Zhong B., Wu S. and Luo H. Construction risk knowledge management in BIM using ontology and semantic web technology. Safety Science, 87:
202-213, 2016.

[13] Wang H. and Boukamp F. Ontology-based representation and reasoning framework for supporting job hazard analysis. J.Comput.Civ.Eng., 25(6): 442-456, 2011.

[14] Smith S., Osborne J. and Forde, M. The use of a discrete-event simulation model with Erlang probability distributions in the estimation of earthmoving production. Civ.Eng.Syst., 13(1): 2544, 1996.

[15] Vahdatikhaki F., Langari, S. M., Taher A., El Ammari K. and Hammad A. Enhancing coordination and safety of earthwork equipment operations using Multi-Agent System. Auto. Const., 81: 267-285, 2017.

[16] Taher A., Vahdatikhaki F. and Hammad A. Towards Developing an Ontology for Earthwork Operations. ASCE, 101-108, USA, 2017

[17] Zhou Z., Goh Y. M. and Shen L. Overview and Analysis of Ontology Studies Supporting Development of the Construction Industry. J.Comput.Civ.Eng., 30(1), 2016.

[18] Uschold M. and Gruninger M. Ontologies: Principles, methods, and applications. The knowledge engineering review, 11(2): 93-136, 1996.

[19] Fernández-López M., Gómez-Pérez A. and Juristo N. Methontology: from ontological art towards ontological engineering. AAAI Technical Report SS-97-06, 33-40, 1997.

[20] Noran O. UML vs. IDEF: An Ontology-Oriented Comparative Study in View of Business Modelling. 674-682, 2004.

[21] Baader F. The description logic handbook: Theory, implementation, and applications, Cambridge University Press, 2003.

[22] Krötzsch M., Simancik F. and Horrocks I. A description logic primer. arXiv preprint arXiv: 1201.4089, 2012.

[23] Du H., Dimitrova V., Magee D., Stirling R., Curioni, G., Reeves H., Clarke B. and Cohn A. An ontology of soil properties and processes. 30-37, 2016.

[24] OSHA. Soil classification. On-line: www.osha.gov/pls/oshaweb/owadisp.show_docum ent?p_table=STANDARDS\&p_id=10931, Accessed: 25/4/2018.

[25] OSHA Trenching and Excavation Safety. U.S.Department of Labour, OSHA, 2015.

[26] OSHA Sloping and Benching. On-line: www.osha.gov/pls/oshaweb/owadisp.show_docum ent?p_table=STANDARDS\&p_id=10932, Accessed: 21/06/2018.

[27] Stanford U., Stanford centre for biomedical informatics research; The Protégé ontology editor. On-line: https://protege.stanford.edu/products.php, Accessed: 08/05/2018. 